\title{
Adaptation auditive et localisation
}

\author{
S. MEUNIER et G. CANÉVET
}

Laboratoire de Mécanique et d'Acoustique, CNRS, 31 chemin Joseph-Aiguier, 13402 Marseille cedex 20, France

\begin{abstract}
This paper presents the main results of an experiment on the possible role of adaptation in auditory localization. Measurements are performed in an anechoic room on the apparent azimuth of a brief $4-\mathrm{kHz}$ tone, coming from the side $\left(15^{\circ}\right)$ of the listener, in the presence of a tone at the same frequency coming from the front. This frontal source is turned on first, for a certain amount of time, in order to induce some sort of auditory adaptation; next, a 50-ms tone is presented from the lateral source. It is found that, as the duration of the pre-exposure is increased, the apparent azimuth of the second sound varies under some conditions; in most cases, it is shifted towards the side of the subject, away from the actual position of the source. The same type of effect is demonstrated under earphones. In this case, the adapting signal is a diotic tone, and the adapted signal is a 50-ms dichotic tone with an interaural time difference of $70 \mu \mathrm{s}$. The summation of these tones leads to an interaural level difference, and therefore to a lateralized auditory image. The apparent location of this image also varies with adaptation, as in the first experiment.
\end{abstract}

\section{INTRODUCTION}

L'adaptation auditive est un phénomène sensoriel complexe qui se manifeste dans divers aspects de la perception des sons. Plus généralement, l'adaptation est un caractère commun à tous les systèmes sensoriels. Elle se traduit par une décroissance dans le temps de la réponse du système à une stimulation continue invariable. Après une certaine durée de la stimulation, la réponse se stabilise à un certain niveau d'équilibre. Selon la durée de stimulation nécessaire pour atteindre ce niveau d'équilibre, on définit deux types d'adaptation : l'adaptation à court terme, pour des durées de l'ordre de la centaine de millisecondes (Zwicker, 1965), et l'adaptation à long terme pour des durées de l'ordre de la seconde et plus (Scharf, 1983). Dans le cas de l'audition, on connait assez bien les effets de l'adaptation sur la sonie, et dans une certaine mesure également sur la détection (effet overshoot). Par contre, on ne sait encore que peu de chose sur l'influence de l'adaptation sur la tonie par exemple, ou sur la perception spatiale.

Cet article a justement pour but de présenter des données récentes sur la question du rôle de l'adaptation dans la localisation auditive. Il fait suite à une note de recherche en cours de publication (Canévet \& Meunier, 1994), où l'on trouvera notamment une brève analyse bibliographique sur le sujet. Les conditions expérimentales utilisées pour la présente étude ont été fixées de manière à permettre la comparaison avec les résultats d'une étude théorique antérieure sur la localisation masquée (Canévet, 1985) et d'une étude en cours sur la détection d'incréments (Meunier \& Marchioni, 1992). L'expérience type de localisation sur laquelle nous avons travaillé a consisté à exposer des auditeurs à un son continu d'une certaine durée, dans le but de produire un certain degré d'adaptation auditive. A ce son était ensuite ajouté un son bref de direction différente; l'objectif étant de mesurer l'azimut apparent du son bref en fonction de la durée de la pré-exposition au son adaptant. Les expériences de localisation ont ensuite été transposées sous écouteurs. Dans ce cas, les signaux utilisés étaient respectivement un son continu diotique, destiné à provoquer l'adaptation, et un son bref dont la latéralisation était obtenue par différences interaurales de niveau et de temps insérées entre les écouteurs. 


\section{CONDITIONS EXPERIMENTALES DES MESURES DE LOCALISATION}

Pour les mesures de localisation, le sujet était placé au milieu d'une salle anéchoïque d'environ $5 \mathrm{mX} 5 \mathrm{mX} 5 \mathrm{~m}$, sur une chaise munie d'un appui-tête. Les sons étaient émis par deux haut-parleurs de $17 \mathrm{~cm}$ de diamètre, logés dans des enceintes d'environ $125 \mathrm{~cm}^{3}$. Ils étaient disposés respectivement face au sujet, pour le son continu adaptant, et à $15^{\circ}$ sur sa droite pour le son bref à localiser. Les deux haut-parleurs étaient placés à la même hauteur, approximativement celle des yeux des sujets. Les signaux utilisés étaient des sons purs à différentes fréquences et différents niveaux (dont la mesure se faisait à l'aide d'un microphone B\&K d'un demi-pouce, à l'emplacement de la tête du sujet en l'absence de celui-ci). Nous présentons ici les résultats obtenus à $4 \mathrm{kHz}$, pour un niveau de pression acoustique de $60 \mathrm{~dB}$. On a obtenu des résultats analogues à $2 \mathrm{kHz}$; mais l'expérience n'a pas pu être menée à $1 \mathrm{kHz}$ ou $500 \mathrm{~Hz}$ car la localisation devient alors trop difficile à cause de la présence d'indices conflictuels (Canévet, 1985).

Les caractéristiques temporelles des signaux dépendaient de la mesure à effectuer. L'étude préliminaire (Canévet \& Meunier, 1994) avait mis en évidence le rôle très important du déphasage entre les signaux. Nous avons donc, dans une première partie, mesuré l'azimut apparent qu'avait le couple de signaux (central + latéral) lorsqu'ils étaient simultanés, et ce pour différentes valeurs de leurs phases relatives. Les deux signaux duraient $50 \mathrm{~ms}$, avec une enveloppe gaussienne de $10 \mathrm{~ms}$ pour l'établissement et l'extinction. Chaque couple était présenté trois fois consécutives pour chaque déphasage. Nous obtenions le déphasage entre voies à l'aide d'une ligne à retard, dont on pouvait faire varier le réglage par pas de 10 ms. Pour la seconde partie, consacrée aux mesures d'adaptation, le signal latéral était le même que cidessus. Par contre, le signal frontal avait les mêmes caractéristiques transitoires, mais une durée variable : il était présenté un certain temps dT avant le signal latéral, et s'arrétait $500 \mathrm{~ms}$ après lui. La durée de la préexposition dT est la variable de l'expérience. Nous avons testé les dix valeurs suivantes, exprimées en ms : $20,80,150,250,500,800,1200,2000,5000$ et 10000 .

Les mesures d'azimut se faisaient par estimation directe. Pour aider les sujets dans leurs estimations, nous avions disposé une série d'étiquettes en arc de cercle dans la chambre sourde, de $270^{\circ}$ (gauche du sujet) jusqu'à $90^{\circ}$ (droite), à intervalle de $15^{\circ}$. Les résultats présentés ici sont ceux de cinq sujets expérimentés, ayant tous une audition normale.

\section{RESULTATS DES MESURES DE LOCALISATION}

Nous avons présenté sur la Fig. 1 les moyennes des estimations des cinq sujets pour la localisation des sons brefs simultanés. Quatre sujets ont fait la mesure quatre fois; le cinquième deux fois. Chaque point du graphe représente donc la moyenne de dix-huit estimations. Les symboles carrés sur la gauche de la Fig. 1 servent à rappeler l'azimut réel des haut-parleurs. Suivant le déphasage introduit entre les signaux d'alimentation des haut-parleurs, on voit que l'azimut apparent de l'image résultante peut varier entre $+60^{\circ}$ et $-60^{\circ}$, suivant les sujets.

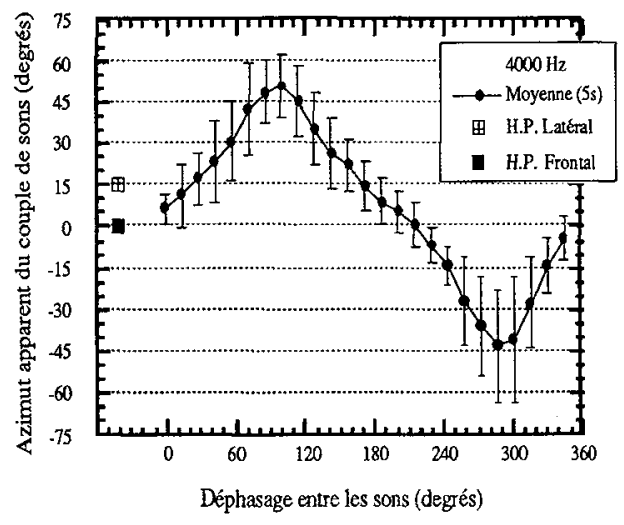

Figure 1. Position apparente de la source viruelle créée par deux sources réelles en fonction de leurs phases relatives à l'émission

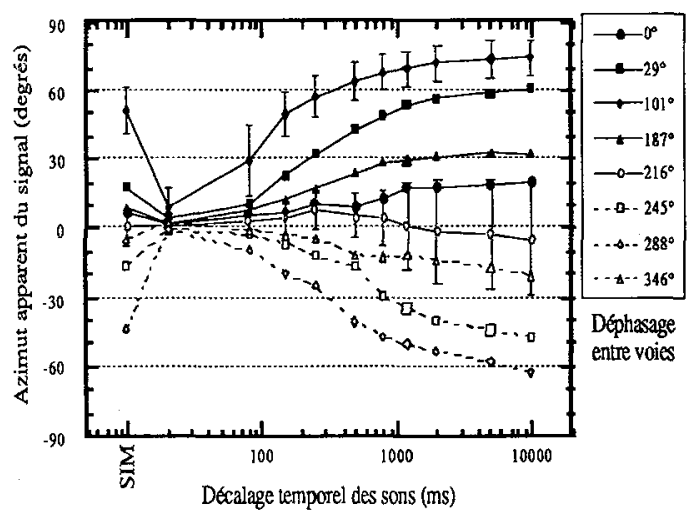

Figure 2. Effet de la pré-exposition sur l'azimut apparent du son latéral, pour diverses valeurs du déphasage entre les sons 
Les résultats de la seconde partie de l'expérience (adaptation en fonction du déphasage) apparaissent sur la Fig. 2. Cette figure appelle plusieurs commentaires. Tout d'abord, les points à la verticale de l'abscisse de $10 \mathrm{~ms}$ (notée SIM, de "simultané") correspondent en fait aux mesures sans décalage, c'est-àdire qu'ils sont tirés de la Fig. 1 pour la série de déphasages indiqués dans le cadre de droite. Nous les avons reliés aux points d'abscisse $20 \mathrm{~ms}$ sur la Fig. 2 pour montrer le recentrage des images obtenu pour les courtes valeurs de pré-exposition. Ce recentrage est sans doute dû à l'effet d'antériorité. Mais la partie la plus intéressante se situe au-delà de 100 à $200 \mathrm{~ms}$ environ, quand l'effet d'antériorité n'agit plus. On observe que, pour certains déphasages, l'azimut apparent du signal latéral se déplace progressivement sur le côté à mesure que le décalage temporel augmente. Etant donné que le champ acoustique sur les oreilles des sujets, lors de la présentation du signal latéral, ne change pas avec le décalage temporel (nous l'avons vérifié par des mesures physiques simultanées), on ne peut attribuer qu'à un effet d'adaptation cette dérive des azimuts apparents. Il est important de noter en plus que l'on peut obtenir, après pré-exposition de plusieurs secondes, des azimuts nettement supérieurs aux azimuts obtenus dans le cas de signaux simultanés pour le déphasage correspondant. On remarquera aussi que les conditions pour lesquels l'azimut ne varie pas, ou peu, correspondent à des déphasages pour lesquels les deux signaux émis simultanément sont localisés au centre. Enfin, la variabilité interindividuelle peut être très forte. Les écarts types,présentés seulement pour deux conditions (déphasages de $101^{\circ}$ et $216^{\circ}$ ), peuvent atteindre $30^{\circ}$ d'angle. Cela traduit probablement une variabilité des effets d'adaptation. Mais en partie cela tient aussi au fait que le phénomène observé est très sensible au déphasage acoustique entre les sons. Or nous fixions ce déphasage avant l'expérience, en un point qui devait être ensuite au centre de la tête du sujet, mais la mesure était faite en son absence. Donc il y a une variabilité inévitable, d'un sujet à l'autre, dans sa position face aux haut-parleurs. Pour éviter cette variabilité supplémentaire, nous avons reproduit à l'aide d'écouteurs une expérience qui simulait au mieux celle que nous venons de décrire.

\section{CONDITIONS EXPERIMENTALES DES MESURES DE LATERALISATION}

Le son frontal était ici remplacé par un signal diotique, obtenu simplement par alimentation en parallèle des écouteurs. Le son latéral résultait un signal dichotique, provenant du même générateur que le signal diotique mais avec une différence interaurale de temps d'arrivée $(70 \mu \mathrm{s})$ créée par une ligne à retard montée en série avec l'écouteur gauche. Enfin, la différence de phase entre sons était produite par une ligne à retard, placée avant les portes de découpage des sons, donc équivalente à un déphaseur. Enfin, les deux voies (diotique et dichotique) étaient additionnées par un sommateur électronique avant d'être connectées aux écouteurs.

Les mesures ont été faites dans une cabine audiométrique, pour des sons à $4 \mathrm{kHz}$, présentés à 65 $\mathrm{dB}$ de niveau de pression sur chaque écouteur. Il y avait deux parties aussi dans cette expérience. La première partie était consacrée à une mesure de latéralisation (localisation intracrânienne). Les conditions de présentation des signaux étaient identiques à celles de la localisation. Pour indiquer la position apparente de l'image résultante, le sujet disposait d'un graphique apparaissant sur son terminal de dialogue (VT 320 de DEC) sous la forme d'une ligne graduée de -5 à +5 , correspondant respectivement à l'oreille gauche et l'oreille droite, la valeur 0 représentant le centre de la tête. Lorsque cette première mesure était terminée, il y avait une pause de plusieurs minutes. Puis l'on commençait la mesure de l'adaptation. Le son frontal (diotique) continu était mis en route $5 \mathrm{~s}$ avant la présentation d'un premier triplet de sons latérauxde $50 \mathrm{~ms}$. Le sujet devait alors juger la position apparente de ces sons latéraux. Puis un nouveau triplet de sons était présenté, et ainsi de suite. Le déphasage entre voies était sélectionné au hasard entre deux présentations successives, et modifié par programmation. Les auditeurs qui ont participé à cette expérience étaient au nombre de cinq; quatre d'entre eux sont les mêmes que ceux de l'expérience précédente.

\section{RESULTATS DES MESURES DE LATERALISATION}

La combinaison des signaux diotique et dichotique produit des niveaux généralement différent sur les deux écouteurs (fonction du déphasage entre voies). Concrètement, la différence interaurale de niveau varie de $-25 \mathrm{~dB}$ (gauche plus intense) à $+25 \mathrm{~dB}$, (droite plus intense) lorsque le déphasage effectue un cycle complet de $360^{\circ}$. La position apparente de l'image intracrânienne passe donc d'une oreille à l'autre au cours du même cycle. C'est ce que montre la Fig. 3 (cercles blancs pour les sons brefs). Dans le cas du son diotique continu, deux effets intéressants se produisent. Le plus frappant est sans doute l'apparition d'images dédoublées pour des valeurs de déphasage allant de $90^{\circ}$ à $180^{\circ}$ environ. Les sujets ne devant donner qu'une seule réponse, on obtient une distribution de points qui sont concentrés en partie à gauche, en partie à droite et le reste au centre. Les écarts types de la Fig. 4 (autour des cercles noirs) évoquent bien cette dispersion. La cause de ce dédoublement d'images auditives n'est pas encore complètement élucidée, mais il se produit dans les cas où le niveau moyen est le plus faible sur les oreilles. 


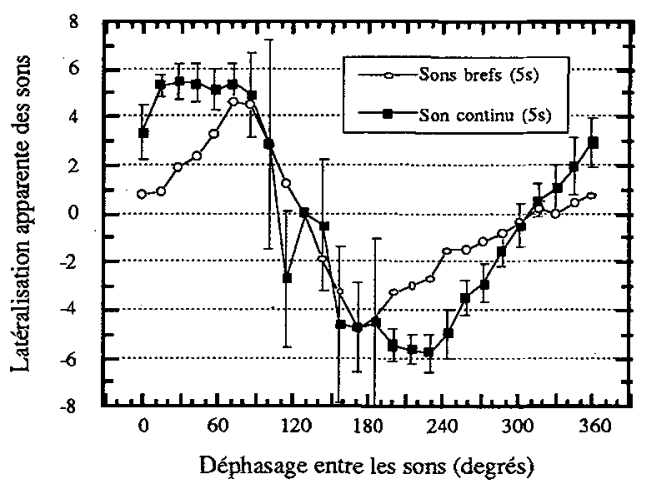

Figure 3. Comparaison des positions apparentes de sons brefs, avec adaptation (cercles noirs) et sans adaptation (cercles blancs)

La seconde observation que l'on peut faire est que, sauf dans la zone à dédoublement, les images sont presque toutes déportées sur le côté, comme dans le cas de la localisation en champ libre. Ceci est surtout net pour les déphasages de $0^{\circ}$ à $90^{\circ}$ par exemple, où les sons se trouvent localisés sur le côté droit (jugement moyen à +5 environ) donc au-delà de ce que l'on observe sans adaptation. Le même effet se retrouve dans l'intervalle de $180^{\circ}$ à $360^{\circ}$, si l'on excepte la zone où les jugements sont centrés pour les deux types de signaux (déphasages au voisinage de $310^{\circ}$ ). De plus on voit bien que le déplacement des jugements augmente bien vers la gauche (de $200^{\circ}$ à $300^{\circ}$ ) ou la droite (au-đelà de $330^{\circ}$ ) suivant la position de référence des images sans adaptation.

\section{DISCUSSION ET CONCLUSION}

La cohérence des résultats permet maintenant d'avancer des conclusions relativement claires sur l'effet que nous avons étudié. Lorsqu'un signal acoustique présenté par écouteurs produit, à cause d'une différence interaurale de niveau, un son décentré dans l'espace subjectif interaural, une pré-exposition diotique au même signal provoque une dérive sur le côté du son décentré. C'est le même phénomène qui se produit dans l'expérience de localisation décrite aux $\$ 2$ et $\$ 3$, puisque la position apparente latérale des sons est là aussi produite par des différences interaurales de niveau résultant de l'interférence et de la diffraction des signaux acoustiques (Canévet, 1985). Dans le cas d'une pré-exposition continue, l'image s'éloigne d'autant plus vers le côté qu'elle est déjà plus éloignée du centre sans pré-exposition. Cette remarque ne tient cependant que pour des durées de pré-exposition suffisamment longues (environ $200 \mathrm{~ms}$ dans les conditions de la présente expérience) pour être au-delà de la zone d'influence de l'effet d'antériorité.

Bien que ces résultats soient à confirmer par une série d'exprériences complémentaires, on peut probablement avancer l'hypothèse que la base des effets observés est une adaptation auditive au son continu, analogue à celle que l'on a trouvée dans les expériences de détection d'incrément. Dans le cas de la perception d'espace, tout se passe comme si la différence interaurale de sonie du signal bref augmentait avec la durée de la pré-adaptation. Cette augmentation étant progressive, elle se traduit par un glissement également progressif de l'image auditive correspondant à ce signal. Dans le cas de l'expérience par écouteurs, le son continu étant maintenu pendant toute la durée des mesures, l'état d'adaptation est maximal et les jugements de position correspondent à une valeur asymptotique du déplacement.

\section{BIBLIOGRAPHIE}

Canévet G. Aspects physiques de la détection et de la localisation masquées. Acustica, 1985, 57, 122-132. Canévet G., Meunier S. Auditory adaptation and lateralization. Acustica, 1994 (sous presse).

Meunier S., Marchioni A. Détection d'un incrément d'intensité à différents niveaux et différentes fréquences. 2ème Congrès Français d'Acoustique, Arcachon, 1992, C1-213/216.

Scharf B. Loudness adaptation. In "Hearing Research and Theory", vol 2, J. V. Tobias \& E. D. Schubert Eds., Academic Press, New York, 1983.

Zwicker E. Temporal effects in simultaneous masking by white-noise bursts. J. Acoust. Soc. Amer., $1965,37,653-663$. 